\title{
Miniaturized rigid probe driver with haptic loop control for neurosurgical interventions
}

\author{
Danilo De Lorenzo, Rudy Manganelli, Ilya Dyagilev, Alessandro Formaglio, Elena De Momi, \\ Domenico Prattichizzo, Member, IEEE, Moshe Shoham, Member, IEEE, and Giancarlo Ferrigno
}

\begin{abstract}
During neurosurgical "keyhole" procedures (i.e. functional neurosurgery and biopsies), linear probes are inserted into the brain toward selected targets. Recently, robotic systems have been introduced to help the surgeon to improve the operation accuracy and safety while simultaneously reducing the invasiveness of the procedure. In this paper, an innovative miniaturized linear actuator for neurosurgical probe insertion, with a remote master device, is presented. The linear actuator, with three piezo-motors that allow the movement of the probe, is equipped with a position sensor and with a force sensor in order to provide the surgeon with haptic feedback. The haptic master drives the linear actuator and reproduces and amplifies the contact forces between the surgical tool and the brain tissue. The results of two different force sensing modalities are compared. First tests show the surgical application requirements are satisfied.
\end{abstract}

\section{INTRODUCTION}

$\mathrm{N}$ EUROSURGICAL interventions require accurate insertion of needles inside the brain tissue (e.g. during biopsies, deep brain stimulation, functional neurosurgery). The standard manual procedure requires needle positioning with a manually adjustable frame, rigidly attached to the patient skull and automatically registered with pre-operative images. An insertion guide is mounted on the frame to provide rigid support and guidance for needle insertion [1]. Nevertheless, the frame requires preoperative implantation of head screws under local anaesthesia and it is bulky, cumbersome and needs manual adjustment during surgery. Several robotic systems were developed to help the neurosurgeon during the needle insertion. Robotic semi-active systems are frame-less stereotactic systems since they automatically positions themselves with respect to a target trajectory [2].

During tele-operated robotics, the surgeon operates on a master device that moves the remote surgical tools with or

Manuscript received April 9, 2010. This work was supported in part by the EU community under Grant ROBOCAST 215190 and by Scuola Interpolitecnica di Dottorato, Politecnico di Milano, Torino e Bari, Italy.

D. De Lorenzo, E. De Momi and G. Ferrigno are with the Neuroengineering and Medical Robotics Laboratory, Bioengineering Department, Politecnico di Milano, Milan, Italy (phone: (0039)0223999013; fax: (0039)0223999003; e-mail: danilo.delorenzo@mail.polimi.it,_elena.demomi@polimi.it, giancarlo.ferrigno@polimi.it).

I. Dyagilev and M. Shoham are with Medical Robotics Laboratory at the Mechanical Engineering Department, Technion, Haifa, Israel (e-mail: silyad@techunix.technion.ac.il, shoham@technion.ac.il).

R. Manganelli, A. Formaglio, and D. Prattichizzo are with Department of Information Engineering, University of Siena, Italy ( $\{$ manganelli,formaglio,prattichizzo $\} @$ dii.unisi.it) without force feedback. The main objective of the haptic control design in minimally invasive surgery is to achieve transparency (i.e. the haptic device has to be "transparent" to the surgeon, as if he/she is holding the real surgical instrument) while maintaining stability (i.e., the system does not exhibit vibration or divergent behaviour), under any operating conditions and for any environment [3]-[6]. The force feedback increases the information about the surgical environment, giving the possibility to the user to intraoperatively detect unexpected situations (e.g. a vessel or a pathological tissue) unrecognized on the pre-operative images.

In order to sense forces, two techniques are mainly used. The first estimates the force being applied to the environment by computing the difference between the desired (master) and the actual pose of the slave robot [3]. The reliability of the technique is limited due to the robot dynamics, that often masks the small interacting forces [7]. The second technique is providing the surgical tool with a force sensor. This is difficult to achieve due both to the harsh surgical environment (i. e. biocompatibility, sterilizability and size constraints) and to the difficulties to add sensing capabilities to instruments not designed for this purpose.

The LANS system [8] was built to perform biopsies and neurosurgical interventions by means of a miniature X-ray source. The tool actuator, mounted on a NeuroMate robotic system [9], is equipped with a DC mini-motor which drives a miniature ball screw through a synchronous belt with a load cell that measures contact forces within the brain tissue. The NeuroArm system, which is magnetic resonance compatible, features ultrasonic piezoelectric motors [10] and titanium multi-axis force/torque sensors on the end effector to provide three Degrees of Freedom (DoFs) force feedback, thus providing high-fidelity haptic feedback to the hand controllers. The system is equipped with proper specialized tool sets thus standard surgical probes cannot be employed. Two commercial devices, the NeuroDrive ${ }^{\mathrm{TM}}$ and the AlphaDrive (both by Alpha-Omega, Nazareth, Israel) allow automatic surgical electrode insertion for brain signal recording. These active systems use rotary electromagnetic motors coupled with linear screw slides to perform the needle insertion. They are not useful for frame-less interventions, since they provide only a short stroke $(40 \mathrm{~mm}$ for the Neurodrive), which could not be enough for several interventions (up to $110 \mathrm{~mm}$ of advancement in the brain 
tissue can be necessary) and they are not able to sense forces.

Piezoelectric actuators are now becoming used in medical applications: they are more energy efficient, they do not generate electromagnetic noise and, since magnetic shielding is not necessary, the design can be kept compact. Inchworm linear motors were proposed in literature since they assure high resolution, unlimited travelling and no backlash [11]. They can feature different piezoceramic elements and magnetostrictive actuators [12], which assure high forces and short response time [13].

This paper describes a miniaturized tele-robotic system for neurosurgical intervention with haptic feedback, designed in collaboration with neurosurgeons, as shown in [14]. The system is able to drive standard surgical needles used for brain biopsies with a piezomotor and to provide online force feedback to the surgeon through an haptic interface. During the design phase, small dimensions, sterilizability, adaptability to standard surgical tools and human factor were considered as constraints.

\section{METHODS}

The linear needle insertion is driven by an inchworm type piezomotor. The driver is equipped with an optical linear encoder, which measures the advancement of the needle, and a force sensor, which provides the surgeon with an amplified sense of touch through an haptic interface (master). This mechanism enables the manual servo-assisted insertion of the probe into soft tissues without the loss of kinaesthetic perception.

\section{A. The Linear Actuator (LA)}

The developed linear actuator is an inchworm type piezomotor with a driver and a shaft as a guide for achieving relative motions (Fig. 1). The driver has two clamps (the back clamp, BC, and the front clamp, FC) and one extension element (the pushing device, $\mathrm{P}$ ) resembling the motion of an inchworm. Each element is actuated by multilayer piezoelectric devices (Noliac, Denmark). In order to increase the piezoelement stroke, mechanical amplifiers were used in the $\mathrm{BC}$, the $\mathrm{FC}$ and the $\mathrm{P}$ element (Fig. 2). The gain of the $\mathrm{FC}$ and $\mathrm{P}$ is 6.5 and the gain of the $\mathrm{BC}$ is 5 , due to design constraints. The maximum needle stroke is $110 \mathrm{~mm}$.

The clamping device BC is stationary and fixed on the support frame, while the FC is mobile and it is mounted on the $\mathrm{P}$ element. The surgical probe is hold by the clamping devices.The holding mechanism is designed to support the end part of the probe and the scanning head of an optical linear encoder with a resolution of $4 \mu \mathrm{m}$ (LIK 41, Numerik Jena). A limit switch resets the optical encoder measurements at home position. This mechanism is mounted on sliding unit that provides low friction motion.

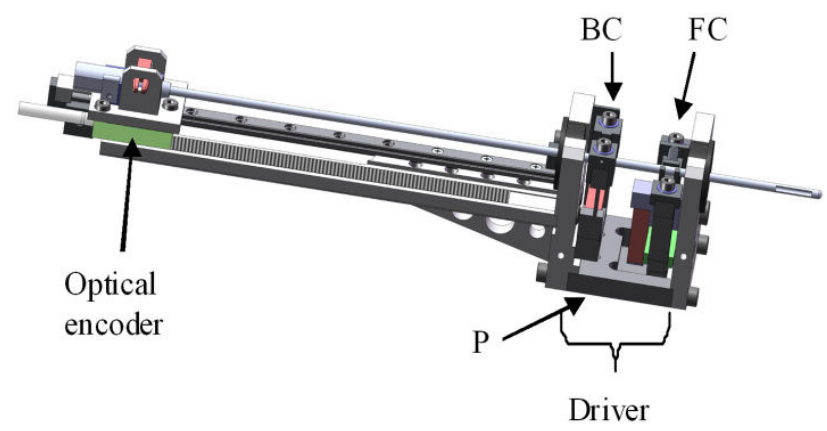

Fig. 1 CAD model of LA. The position of the assembled BC, FC and P is shown.

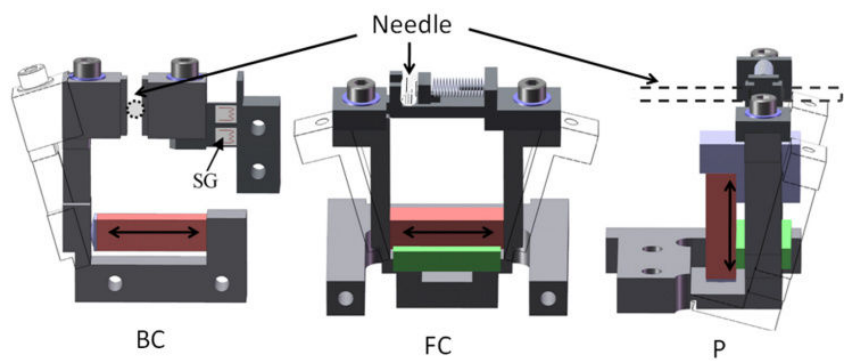

Fig. 2 Mechanical amplifier for BC (on the left), for the FC (in the middle) and for the $\mathrm{P}$ element (on the right). On the $\mathrm{BC}$, the SG position is shwn. The red blocks are the piezo-elements.

The LA allows backward and forward motion of the needle. Two different cycles of opening - closing and pushing - were implemented for the two types of motion: the strokes sequences for the forward motion are reported in Fig. 3 and the corresponding activations of $\mathrm{BC}, \mathrm{FC}$ and $\mathrm{P}$ are reported in Fig. 4. Backward movement cycles can be obtained by similarity. The frequency of actuation is $100 \mathrm{~Hz}$ (10ms per motion cycle). In order to make the probe advance, the needed mechanical steps are six. The change in velocity is achieved linearly increasing or decreasing the voltage applied on the pushing element from 6 to 32V (1023 levels) since the variation in voltage changes the piezoelement stroke amplitude.

\section{B. The force sensor}

The force sensor is made of four strain gauges (SG) in full bridge configuration. In order to identify the maximum strain points where to attach the four SG, the expected strain on the LA component was computed using COSMOSWorks 2007 (SolidWorks, MA, USA) simulating an applied force of $0.01 \mathrm{~N}$ on the BC. The strain gages (Vishay EA-06031CE-350/LE) were placed at maximum strain location $0.5 \mathrm{~mm}$ from the beam support (Fig. 2). The force sensor is capable of sensing only the force exerted along the main axis of the biopsy needle. The SG have a gauge factor of 2.08, $350 \Omega$ resistance and the excitation voltage on the bridge was 10 Volt. The output signal is conditioned (Bandwidth = $1 \mathrm{kHZ}$, Gain = 250). 


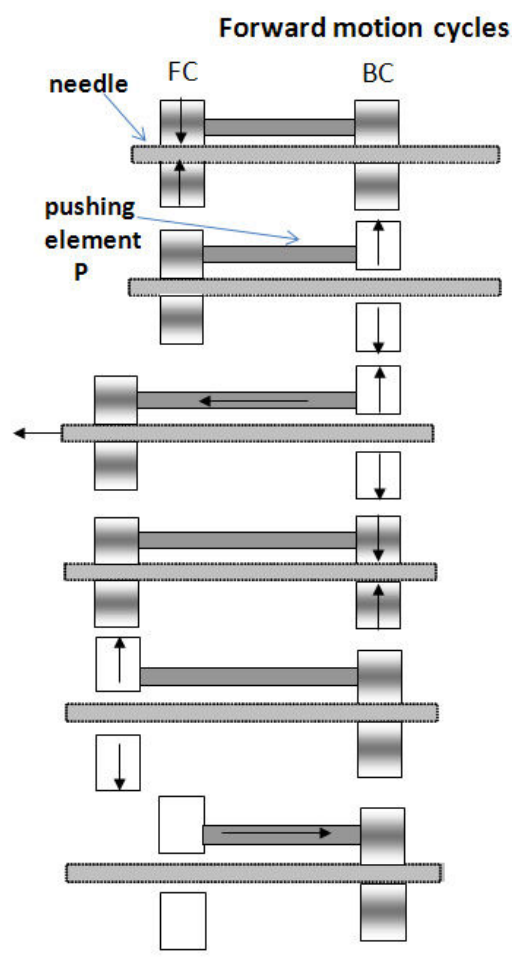

Step1: BC and FC
are close

step:Bcisopen

Step2: BC is open

Step3: the shaft is moved forward

Step4: $B C$ is close

Step5: $\mathrm{FC}$ is open

Step6: FC is moved backward

Fig. 3 Forward motion mechanical cycles.

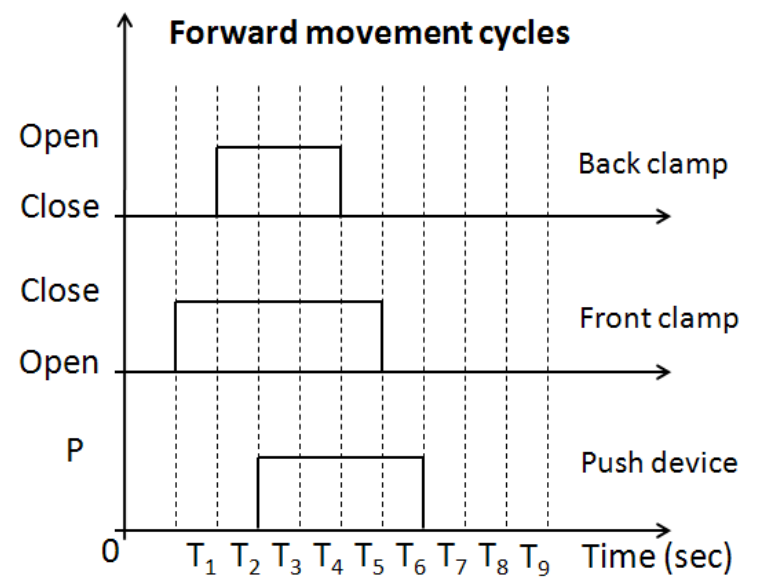

Fig. 4 - The activations for $\mathrm{BC}, \mathrm{FC}$ and $\mathrm{P}$ during forward motion are reported.

\section{The haptic interface}

In order to reproduce the movement of the surgeon during the manual procedure, the motion of the master end-effector was constrained to 1 DoF (Omega 3, Force Dimension, Switzerland). Given the parallel kinematic structure of the Omega, three rigid clamps were applied on the device links (Fig. 5), hence only the $\mathrm{z}$-axis coordinate of the haptic device reference frame is able to move. Other eventual nonzero displacements in the xy-plane (e. g. due to clamp backlash) are neglected. Similarly, the force feedback is rendered only along the $\mathrm{z}$-axis.

In order to assure the stiffness and to preserve the haptic transparency, lightweight aluminium clamps were designed.
Moreover, the original end-effector was replaced by an ergonomic aluminium stick which resembles the real needle. A switch button is used to easily enable/disable the slave probe motion.

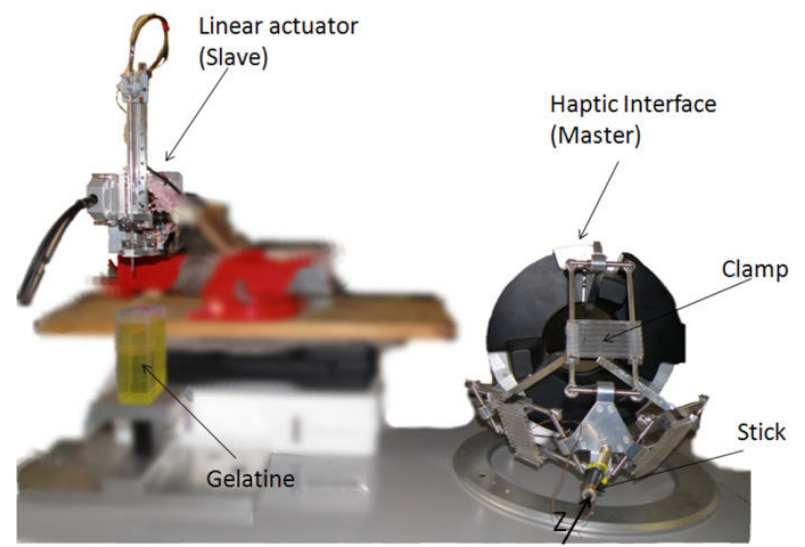

Fig. 5 The overall system. On the haptic interface are visible the clamps used to constrain the Omega device to $1 \mathrm{DoF}$ and the stick.

The Omega3 and the LA are coupled in a master-slave tele-operation architecture [15]. A control strategy was designed such as: the speed of any tool within the patient shall not exceed $2 \mathrm{~mm} / \mathrm{s}$. The force reflection has to be fed back with slow dynamics. Hence, a proportional-derivative (PD) controller was designed in order for the slave endeffector to track the master end-effector motion, filtering out all the frequency contents exceeding a predefined bandwidth. The PD transfer function $C(z)$ is defined as:

$$
C(z)=k_{P}-\frac{z-1}{T_{S} z} k_{D}
$$

where $k_{p}$ is the proportional gain, $k_{d}$ the derivative coefficient and $T_{s}$ is the sampling interval. The control system is represented in Fig. 6:

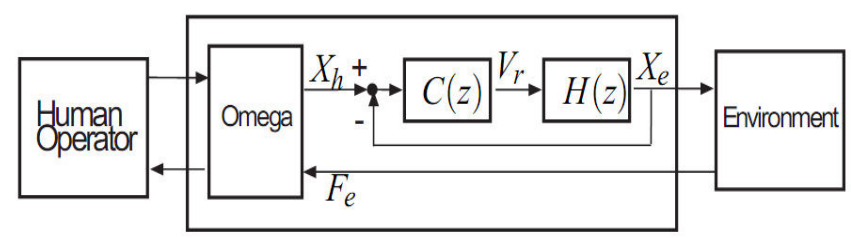

Fig. 6 The master-slave teleoperator system with the PD controller.

where $X_{h}$ and $X_{e}$ are the master (Omega 3) and the slave (LA) end-effector positions respectively, $V_{r}$ is the velocity reference for the slave and $F_{e}$ is the force measured by the slave sensor. $H(z)$ is the transfer function of the slave dynamics.

In order to filter out the noise from the velocity signals, a discrete-time First Order Adaptive Windowing filter [16] was adopted. It minimizes the velocity error variance requiring no trade-off between noise reduction, delay, accuracy, reliability and computational load. As the size $n$ of the filter window has been adapted, the velocity sample $V(k)$ at time instant $k$ is computed as: 


$$
V(k)=\frac{1}{n T_{c}}\left(X_{h}(k)-X_{h}(k-n)\right)
$$

where $X_{h}(k)$ is the master end-effector position at time instant $k$ and $T_{c}$ is the sampling interval.

The communication between the master and the slave is implemented by two serial cables via RS-232 communication protocol. One connection provides position and velocity signals, while the other sends force data. Communication safety is guaranteed by CRC and Timeout Management. The last byte of the package is used to validate the data transmitted. In case of data corruption, the Haptic Controller sends the request again. For the Timeout Management, a package takes about $3 \mathrm{~ms}$ to be delivered, so a timeout of $5 \mathrm{~ms}$ is set to prevent the system to get stuck in case of data loss. In case of timeout error, the haptic controller sends the request again.

\section{Experimental protocol}

Experimental tests were performed in laboratory. A Backlund $(2.1 \mathrm{~mm}$ diameter) brain biopsy needles advancement was tested in air (in order to quantify the noise on the force signal detected during the motion using the force sensor, due to the system assembly) and inside a soft tissue mimicking material. A gelatine sample was prepared as suggested in [17]. An one-axis load cell (AB BOFORS KRK-2) was placed under the gelatine sample in order to acquire the reference force signal. As shown in Fig. 7, the LA was vertical and $6 \mathrm{~cm}$ forward motion was performed. During the needle advancement, the force was measured during the "rest phase" ( $\mathrm{T}_{8}$, of Fig. 4), i.e. when the BC was closed and the FC was open and no pushing occurred.

In order to measure the relaxation time of the gelatine, the needle was manually inserted, the BC closed and the signal acquired. Analyzing the measured signals, the force-time relationship $\mathrm{F}(\mathrm{t})$ is the following:

$$
F(t)[N]=-2.81 \cdot e^{-t[s] / 3}-0.11 \cdot e^{-t[s] / 5000}
$$

$\tau_{1}$ equal to $3 \mathrm{~s}$ means that, after the needle advancement during $\mathrm{T}_{3}$ and $\mathrm{T}_{4}$, the measurement error is at maximum $1 \mathrm{mN}$ at the end of $\mathrm{T}_{8}$, which lasts $1 \mathrm{~ms}$ (at $1 \mathrm{kHz}$ ).

Two force sensing modes were proposed: measuring the force signal from the force sensors (described in Section B) and estimating the force signal computing the difference between the reference velocity for the slave $\left(V_{r}\right)$ and the desired one, $\dot{X}_{h}$ (derivative of $X_{h}$, from the master, Fig. 6).

In order to identify the relationship between the voltage level on the $\mathrm{P}$ piezoelement and the resulting velocity of the needle $\left(\dot{X}_{e}\right)$, tests were performed moving the needle in air. Ten voltage levels were considered (8-32V) averaging 4 repeated measurements. The result was used to compute the velocity reference $V_{r}$.

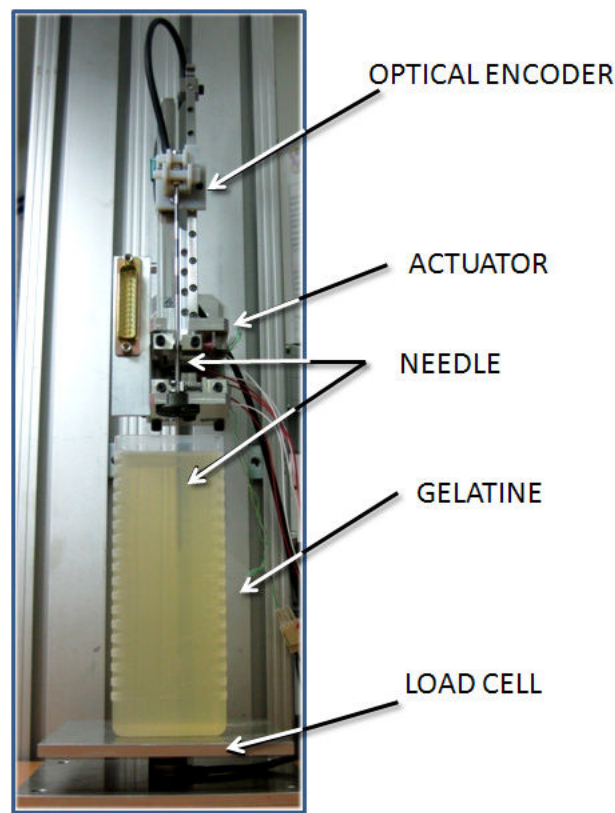

Fig. 7 - Experimental setup for the needle insertion into gelatine. The load cell was positioned at the bottom of the gelatine sample.

Finally, the difference $V_{e}$ between the desired (master) velocity and the reference velocity $\left(\dot{X}_{h}-V_{r}\right)$ was used as an estimation of the force acting on the surgical needle.

Two different tests were performed. In the first test the needle was moved inside the gelatine for 5 trials, using the control algorithm provided in (1) with a fixed velocity value $(0.5,0.8,1,1.3,2 \mathrm{~mm} / \mathrm{s})$.

Signals acquired from the load cell, from the force sensors and the velocity error $\left(V_{e}\right)$ were synchronized and $2 \mathrm{~Hz}$ lowpass filtered. The force sensor signal and the velocity error signal were scaled using least square minimization algorithm (Levenberg-Marquardt). Scale factor and offset were therefore computed. For this test, the root mean square error (RMSE) between the force signal and the load cell was evaluated. Also, the RMSE between $V_{e}$ and the load cell was calculated.

In the second test, the needle was moved in a two layer sample of gelatine, where the first layer was kept at half the concentration of the second. The purpose of this trial was to evaluate the possibility to feel the puncturing between two different kind of tissue. 5 signals were acquired moving the needle at $2 \mathrm{~mm} / \mathrm{s}$ and the RMSE computed.

\section{RESULTS}

Force signals measurements showed drift due to room temperature chaging during the experiments (about $0.1 \mathrm{mN} /$ $\min$.).

As shown in Fig. 8, during $\mathrm{T}_{8}$ of the forward motion, the force signal is stable (oscillations are negligible). Therefore it proved to be a good sampling time frame.

The maximum exerted force measured using a dynamometer, actuating the LA at $100 \mathrm{~Hz}$, was $1 \mathrm{~N}$. 


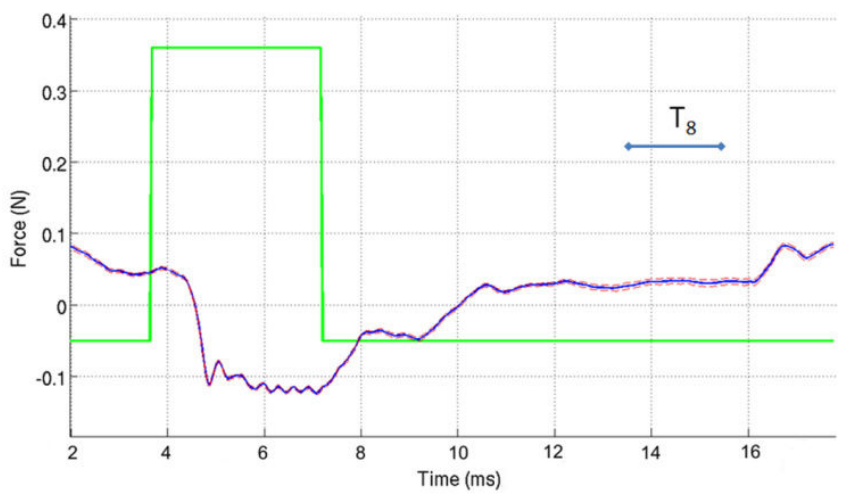

Fig. 8 Force signal acquired from $\mathrm{BC}$ during 50 cycles of probe advancement (forward motion) in the air (mean signal \pm standard deviation signal). The square wave represents the $\mathrm{BC}$ activation signal.

The relationship between the voltage level on the $\mathrm{P}$ and the needle velocity was found to be linear (Fig. 9) . Under 200 (6V) the linear actuator was always moving at the same velocity because of gravity.

As shown in Fig.10, median values of the RMSE are below $0.1 \mathrm{~N}$ for all the tests performed. Even if the three median values are similar, the force sensor performed slightly better $(0.087 \mathrm{~N}$ vs. $0.091 \mathrm{~N})$.

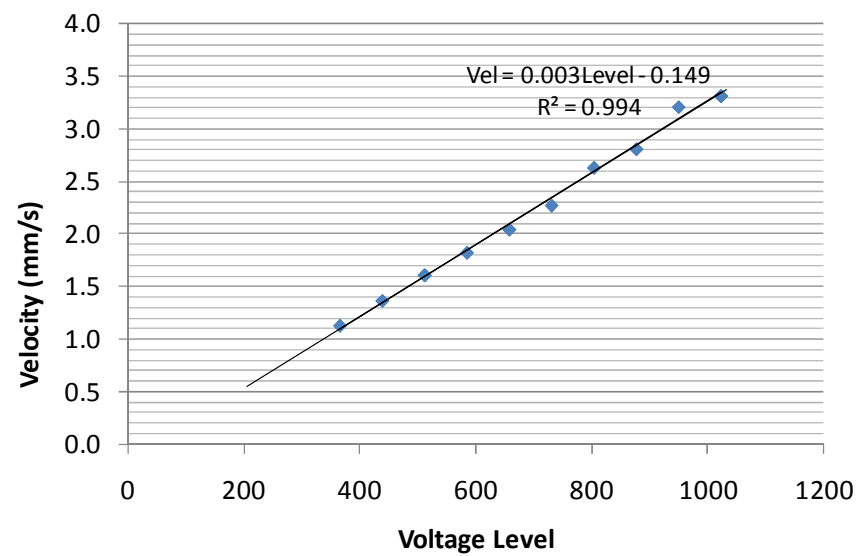

Fig. 9 - Relationship between voltage level and velocity of the needle. The voltage level range is between 0 and 1023 corresponding to 0 and $32 \mathrm{~V}$.

In Fig. 11 the signals from the strain gages and the signal acquired with the load cell are reported. Before the puncturing of the tissue, the force increases up to $0.6 \mathrm{~N}$, which is the mean value found in literature for brain puncturing forces [18],[19].

\section{DISCUSSION}

The paper presents a tele-operated robotic system for neurosurgical application with haptic feedback. A Force sensing device to be used in neurosurgery was presented in [8], but numerical results are not clearly shown.

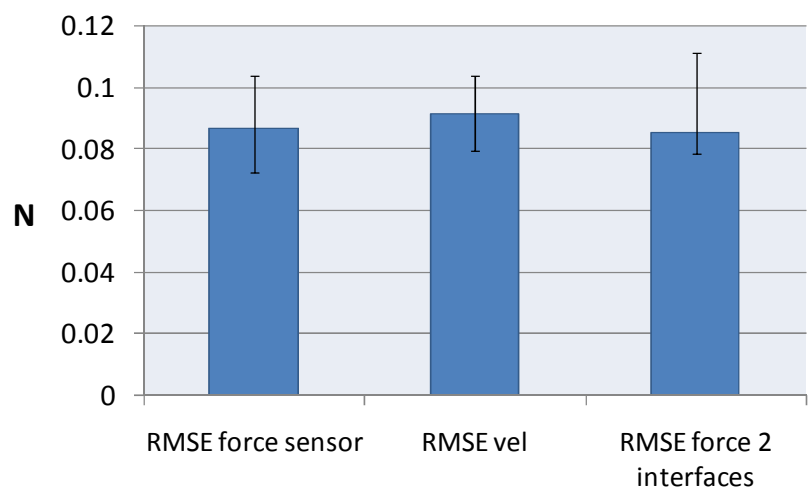

Fig. $10-$ RMSE (median, $25^{\text {th }}$ and $75^{\text {th }}$ percentile) between the force sensor and the load cell (RMSE force sensor and RMSE force 2 interfaces) and between the velocity error and the load cell (RMSE vel).

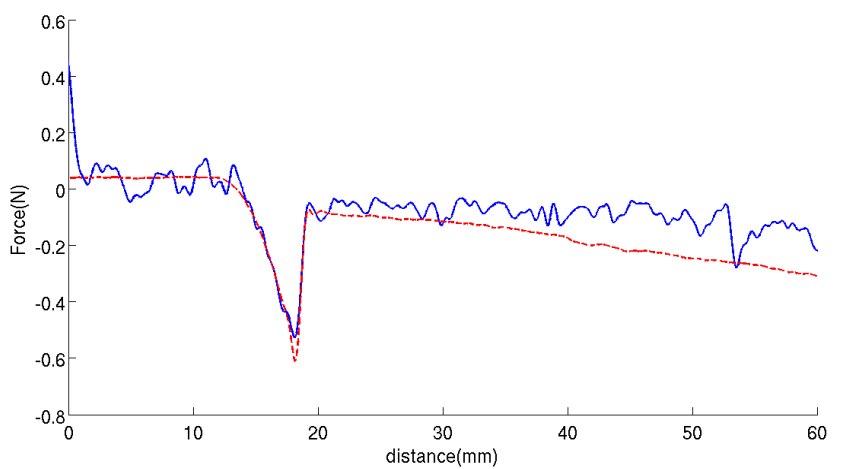

Fig. 11 Force signal detected by the load cell (dashed line) and from the force sensor (solid line) during probe advancement in the gelatine. The force increase due to the probe entering the gelatine is visible $(15-19 \mathrm{~mm})$, together with the force sudden decrease $(19-20 \mathrm{~mm})$, due to gelatine puncturing.

The proposed system is able to sense small forces (up to $1 \mathrm{~N})$ and can therefore be used for surgical application. As shown, the mounted force sensor is able to accurately measure the brain-mimicking material resistance, especially during the puncturing phase, when the needle is cutting the outer layer of the soft material. Therefore the system allows the user to reliably detect unexpected structures along the needle path toward the target (e.g. vessels). The change in force can be also amplified. When the two-layer gelatin is used, the force sensor allows detecting the puncturing between the two interfaces slightly better, with no significant difference with respect to air/gelatine interface.

The calibration test proved there is a linear relationship $(\mathrm{R}$ $=0.99$ ) between the voltage applied on the pushing piezoelement and the needle velocity. Therefore the error between the desired position and the actual one was used as a force measurement.

The two sensing techniques (force sensor and velocity error) did not show any significant difference in terms of RMSE with respect to the load cell measurements. The gelatine resistance to the probe advancement could be therefore estimated avoiding any force sensor that can interfere with the actuator design. Further investigations will regard force sensing estimation with real brain tissue samples. 
The motion of the master end-effector has been mechanically constrained to $1 \mathrm{DoF}$. It would be also possible to constrain the surgeon to move along a straight line using force feedback, but this feature would increase the computational load of the control software, it would not be able to guarantee high structural stiffness and it would probably interfere with measured forces from the brain.

Small noise, due to mechanical friction, could prevent the brain resistance below $0.1 \mathrm{~N}$ to be detected. The friction is mainly due to the small vibration of the needle during the clamping shocks. A damping mechanism (mechanical or electrical) should improve the force sensing capabilities. Since a significant drift was observed in the acquisitions due to room temperature changes, drift compensation implies it is necessary to recalibrate before every needle insertion in the brain tissue

The developed system proved to be embedded into a proper haptic loop. The $2 \mathrm{~Hz}$ low-pass filter on the force signal allows avoiding the surgeon to introduce instability in the outer loop and cancelling physiological tremor which has typical frequency content from 10 to $30 \mathrm{~Hz}$ [20]. The surgeon can therefore benefit of the force sensation during the probe advancement into the brain tissue. However, recently studies suggest that the role of haptic feedback can vary for novice and experienced robot-assisted surgeons [21]. So, further investigation will regard surgeon test and training.

It should be mentioned that the miniaturization of the LA can be achieved by replacing the linear encoder which is the longest component of the linear drive by a rotary encoder thus compacting the design to about one third of its current length into proximally $50 \mathrm{~mm}$ length, becoming the smallest surgical needle driver with force sensing.

Also, the proposed solution allows easy sterilizability and wide range of applications since the force sensor is not directly attached to the surgical needle, the positioning accuracy is $5 \mu \mathrm{m}$ and can support different types of standard surgical needles.

\section{CONCLUSION}

The paper presents the design and first tests on a novel miniaturized LA integrated with an haptic interface to be used in several neurosurgical applications. The system could be reused in other surgical applications where the clinical requirements meet the system technical specifications (i.e. soft tissue needle insertion).

\section{REFERENCES}

[1] R. L. Galloway, R.J. Maciunas, "Stereotactic neurosurgery,". Crit Rev Biomed Eng, vol. 18(3), pp. 181-205, 1990

[2] B. Davies, S. Starkie; S. J. Harris, E. Agterhuis, V. Paul and L. M. Auer, "Neurobot: a special-purpose robot for neurosurgery," in Proc. Int. Conf. on Robotics and Automation, pp. 410-414, 2000.

[3] B. Hannaford, "A design framework for teleoperators with kinesthetic feedback," IEEE transactions on Robotics and Automation, vol. 5(4), pp. 426-434, 1989.

[4] J. Ryu and D. Kwon, "A novel adaptive bilateral control scheme using similar closed-loop dynamic characteristics of master/slave manipulators," Journal of Robotic Systems, vol. 18(9), pp. 533-543, 2001.
[5] R. Anderson and M. Spong, "Asymptotic stability for force reflecting teleoperators with time delay," The International Journal of Robotics Research, vol. 11(2), p. 135, 1992.

[6] S. Salcudean, "Control for teleoperation and haptic interfaces," Lecture Notes in Control and Information Sciences, pp. 51-66, 1998.

[7] M. Mahvash and A. M. Okamura, "Friction compensation for enhancing transparency of a teleoperator with compliant transmission," IEEE Trans. Robot., vol. 23(6), pp. 1240-1246, 2007.

[8] A. Rossi, A. Trevisani, and V. Zanotto, "A telerobotic haptic system for minimally invasive stereotactic neurosurgery," Int. J. Medical Robotics and Computer Assisted Surgery, vol 1(2), pp 64-75, 2005.

[9] T. R. K. Varma, P. R. Eldridge, A. Forster, S. Fox, N. Fletcher, M Steiger, P. Littlechild, P. Byrne, A. Sinnott, K. Tyler, S. Flintham, "Use of the NeuroMate Stereotactic Robot in a Frameless Mode for Movement Disorder Surgery," Stereotact Funct Neurosurg, vol. 80, pp. 132-135, 2003.

[10] G. R. Sutherland, I. Latour, A.D Greer, "Integrating an image-guided robot with intraoperative MRI: a review of the design and construction of neuroArm," IEEE Eng Med Biol Mag., vol. 27(3), pp.59-65, 2008.

[11] B. Zhang, Z. Q. Zhu, "Design of an inchworm-type linear piezomotor," In International Symposium on Smart Structures and Materials (SPIE), Orlando, Florida, USA, February 1994, vol. 2190 , pp. 528-539.

[12] J. Kim, J. D. Kim, S. B. Choi, "A hybrid inchworm linear motor," Mechatronics, vol. 12 (4) , pp. 525-544, 2002.

[13] J. Li, R. Sedaghati, J. Dargahi, D. Waechter, "Design and development of a new piezoelectric linear inchworm actuator," Mechatronics, vol. 15(6), pp. 651-681, July 2005.

[14] E. De Momi and G. Ferrigno, "Robotic and artificial intelligence for keyhole neurosurgery: the ROBOCAST project, a multimodal autonomous path planner," Proceedings of the Institution of Mechanical Engineers, Part H, Journal of Engineering in Medicine, in press.

[15] J. Semmoloni, R. Manganelli, A. Formaglio, D. Prattichizzo, "Control design issues for a microinvasive neurosurgery teleoperator system," In Proc. IEEE Int. Conf. on Advanced Robotics, Munich, Germany, June 2009.

[16] F. Janabi-Sharifi, V. Hayward, and C. Chen, "Discrete-t ime adaptive windowing for velocity estimation," IEEE Trans. Control Systems Technology, vol. 8(6), pp. 1003-1009, 2000.

[17] T. Parittotokkaporn, L. Frasson, A. Schneider, S. E. Huq, B. Davies, P. Degenaar, J. Biesenack and F. M. R. Baena, "Soft tissue traversal with zero net force: feasibility study of a biologically inspired design based on reciprocal motion," In Proceedings of the 2008 IEEE International Conference on Robotics and Biomimetics, Bangkok, Thailand, 22-25 February 2008, pp. 80-85.

[18] A. Wittek, T. Dutta-Roy, Z. Taylor, A. Horton, T. Washio, K Chinzei, K. Miller, "Subject-specific non-linear biomechanical model of needle insertion into brain," Comput Methods Biomech Biomed Engin., vol. 11(2), pp.135-46, 2008.

[19] L. Frasson, T. Parittotokkaporn, A. Schneider, B.L. Davies, J.V. Vincent, S.E. Huq, P. Degenaar, F.M. Baena, "Biologically inspired microtexturing: investigation into the surface topography of nextgeneration neurosurgical probes," in Proc IEEE Eng Med Biol Soc., 2008, pp. 5611-4

[20] C. N. Riviere, J. Gangloff, M. de Mathelin, "Robotic Compensation of Biological Motion to Enhance Surgical Accuracy," Proceedings of the IEEE, vol. 94, no. 9, pp. 1705-1716, Sep. 2006, DOI: 10.1109/JPROC.2006.880722.

[21] C. R. Wagner and R. D. Howe, "Force feedback benefit depends on experience in multiple degree of freedom robotic surgery task," IEEE Trans. Robot., vol. 23(6), pp. 1235-1240, 2007. 